\title{
Perceptual Supports for One-to-One Correspondence in the Conservation of Number
}

\author{
Patricia H. Miller and Richard F. West \\ University of Michigan
}

\begin{abstract}
This study compared provoked and spontaneous one-to-one correspondence along with two tasks having more perceptual support for correspondence. These four levels of correspondence appeared in a conservation of number condition and two conservation-related conditions (a partially static array or a static array). There were two sets of stimuli (toy animals or corks) in each condition. In opposition to Piaget's predictions, the four levels of correspondence did not differ in difficulty for kindergarteners. There was a strong order effect $(p<.001)$ in the direction of increasing conservation over the eight trials. There was some evidence that the standard conservation condition was easier than the other two conditions. Implications for Piaget's theory are discussed.
\end{abstract}

If each object in one group can be paired with an object in a second group and there are no objects left over, the two groups have the same number of objects. This concept, called one-to-one correspondence, is basic to the development of conservation, according to Piaget (1952). Eventually, children can maintain the correspondence despite changes in physical arrangement. In spite of the importance Piaget attaches to correspondence, very little is known about this concept. This is especially surprising when one considers the sizable literature on other abilities related to conservation, e.g., compensation, reversibility.

Piaget (1952) claims that the closer the one-to-one correspondence between the elements of two sets, the more likely the child is to notice and maintain the equivalence. Thus, he states that provoked correspondence, e.g., sets of flowers and vases, eggs and egg cups, is easier than spontaneous correspondence, e.g., identical chips which have no natural relationship. However, he offers no evidence for this claim. The few studies which have examined this claim are marred by methodological problems. Dodwell (1960) found that provoked correspondence was easier than spontaneous, but the testing was different for the two. In the provoked task, eggs were bunched up and the children were asked whether there were the same number of eggs and egg cups. In the spontaneous

This study was supported by University of Michigan Rackham Faculty Research Grant 984-360222 to the senior author. The authors are grateful to Mitchell School for participating in the study. Requests for reprints should be sent to Patricia H. Miller, Department of Psychology, University of Michigan, Ann Arbor, Michigan 48109. 
correspondence task, poker chips were spread out and the children were asked, "Which row has more pieces in it now?" The difficulty of the spontaneous correspondence could easily be due to the question asked. Hood (1962) found the same results, but the lack of description of materials, questions, transformations, etc., makes it impossible to evaluate this outcome. Rothenberg (1969) found no difference in performance on the two kinds of correspondence tasks, but the materials for the provoked correspondence (matched pairs of toys) may have been more distractible than the materials for the spontaneous correspondence (homogeneous styrofoam blocks). Clearly, if any conclusion is to be made about the difficulty of the two tasks, it is necessary to equate the tasks on all dimensions except the emphasis on correspondence.

The emphasis on correspondence which Piaget believes underlies superior performance with provoked correspondence can be extended even further. Spontaneous correspondence uses either arbitrary correspondence, i.e., any object in one group can be paired with any object in the other group; or spatial correspondence, i.e., the left-most object in one line goes with the left-most object in the other line. In provoked correspondence, the materials "suggest" togetherness. To increase the salience of correspondence even more, there could be unique pairs, e.g., two blue chips, two red chips. In this way the link between specific pairs is emphasized. In a general study of perceptual supports for correspondence, Miller, Heldmeyer, and Miller (1975) found that this type of correspondence facilitated conservation slightly more than did the spontaneous correspondence task. Although Whiteman and Peisach (1970) did not find facilitation of conservation when there were unique pairs, they did find facilitation when the unique pairs were joined by guidelines. These guidelines create an even stronger perceptual link between corresponding pairs. Piaget and Morf (in Piaget \& Inhelder, 1971) found that guidelines were helpful when there were four pairs of objects but were of little help when there were 12 pairs.

The present study examined these four correspondence tasks. The purpose was to determine whether conservation tasks with varying degrees of emphasis on correspondence actually cause different levels of performance as Piaget suggests. As the saliency of correspondence is increased, it should become easier to keep track of corresponding pairs and maintain the correspondence over the transformation. The four types of correspondence were presented with two different sets of materials as a test for generality. Unlike previous research, the tests for the different types of correspondence were as similar as possible.

A between-subject variable was three conditions which differed in the degree to which the presentation of stimuli emphasized correspondence. Condition 1 was the standard procedure for assessing conservation in which two rows of objects of equal length were set up, the child was 
questioned about their equality, and then one row was spread out. In Condition 2, when the two rows of equal numbers were set up, one of the rows was already longer. It should be more difficult for the child to attend to correspondence in this condition because he never sees the corresponding pairs aligned with one object above the other. Thus, fewer children were expected to express a belief that the rows have equal numbers. Condition 3 was the same as Condition 2, except that the child did not see the pairs set down; he was shown rows of objects which were glued down. Even less conservation was expected here than in Condition 2, because there was neither an initial alignment of each pair (as in Condition 1), nor a setting out of objects pair by pair (as in Conditions 1 and 2). In order to use correspondence in Condition 3, the child himself must initiate a search for corresponding pairs, ignoring differences in the lengths of the rows. Beilin (1969) found that it was more difficult to conserve with a static presentation (like Condition 3 ) than with the standard tests of conservation of area and number.

It should be noted that Conditions 2 and 3 are not conservation tasks as defined by Piaget, i.e., they do not follow the necessary three steps $\left(A=B, B \rightarrow B^{\prime}, A\right.$ ? B). However, they do require a judgment of equality in the face of a misleading perceptual cue, i.e., different lengths. They are more accurately described as conservation-related tasks in which quantitative comparisons can be made. They served to test for the generality of any effects of level of supports for correspondence found in the conservation task of Condition 1.

\section{METHOD}

\section{Subjects}

The subjects were 72 kindergarten children (mean age $=5$ years, 10 months) from a predominantly white, middle-class elementary school in Ann Arbor, Michigan. The 35 males and 37 females were randomly assigned to three experimental conditions. Four additional children were rejected because they did not pass all three parts of the verbal pretest.

\section{Design}

Each subject received eight trials - four with small plastic animals and four with corks. Within each stimulus set there were four levels of emphasis on one-to-one correspondence. For half of the children the four animal trials came first and for half the four cork trials were first. Within each stimulus set there were four orders of the four types of trials (levels of emphasis) so that each trial was in each of the four positions an equal number of times. For a particular child, the order of the four trials was the same for the animal set and the cork set. This design was 
followed in the three experimental conditions which differed in how the experimenter presented the stimuli.

\section{Materials}

The materials were plastic animals (about $2.5 \mathrm{~cm}$ wide and $4 \mathrm{~cm}$ long), paper box "cages" ( $4 \mathrm{~cm}$ wide and $5 \mathrm{~cm}$ long), corks $2.5 \mathrm{~cm}$ in diameter and $3 \mathrm{~cm}$ tall), bottles $(2.5 \mathrm{~cm}$ in diameter and $6 \mathrm{~cm}$ tall), and red yarn.

The four types (levels) of trials were as follows, listed in order of decreasing emphasis on correspondence. The correspondence cues are in parentheses.

A. Seven pairs of seven different animals or corks of seven different colors, with yarn connecting each pair (spatial position, provoked relationship, appearance of objects, guidelines).

B. Seven pairs of seven different animals or corks of seven different colors (spatial position, provoked relationship, appearance of objects).

C. Seven identical turtles paired with their identical cages or seven uncolored corks with their bottles. This is a provoked correspondence task (spatial position, provoked relationship).

D. Seven pairs of identical turtles or uncolored corks. This is a spontaneous correspondence task (spatial position).

\section{Procedure}

One male graduate student tested each child individually in a small room at the school. The child was first given a pretest for the verbal terms which would be used in the conservation trials. The experimenter placed two groups of three chips in front of the child and asked, "Is there the same number of chips in this group as in this group (experimenter points), or does one group have more?" (Version 1). The procedure was then repeated with groups of two and four chips, except the question was reversed - "Does one group have more, or is there the same number of chips in this group as this group?" (Version 2).

The pretesting was followed by the eight conservation (or conservationlike) trials. There were three conditions which varied in how the stimuli were presented. Condition 1 followed the standard procedure for testing for conservation of number. Seven pairs of objects were set out, pair by pair, in two horizontal lines of $30 \mathrm{~cm}$. After the child agreed that the lines had the same number of objects, one line was spread out to $48 \mathrm{~cm}$, and the child was again questioned about their equality. The wording of the questions was the same as in the pretest. Versions 1 and 2 alternated from trial to trial. The longer line was nearer the child for one half of the trials and equally often with each version of the conservation question. Children were asked for explanations for their answers. Test materials were removed from the child's view between trials. 
Condition 2 was the same as Condition 1 except that when the pairs of objects were set out, one line was longer than the other. In other words, the child never saw the lines with identical lengths and never saw them being spread out. Each trial began with the end state of the trials in Condition 1 and the conservation question was asked. Condition 3 was the same as Condition 2 except that the child did not see the individual pairs set down; they were glued to cardboard.

\section{Scoring}

There were two types of criteria for conservation: a) a conservation judgment (C), and b) a conservation judgment accompanied by an adequate explanation (EC). A child was credited with a conservation judgment if he believed that the lines had the same number (after the transformation, for Condition 1). Explanations considered to be adequate were: same number (e.g., "They both have seven"), irrelevancy of the transformation or appearance, one-to-one correspondence, no addition or subtraction of objects, reversibility, compensation, and previous equality.

\section{RESULTS}

Since there were no significant sex differences, analyses were done with sexes combined.

An analysis of variance was performed separately on conservation judgments $(C)$ and conservation judgments accompanied by an adequate explanation (EC). The analyses indicated a highly significant effect of order, Trials $1-4$ vs. Trials $5-8, F(1,69)=20.06, p<.001$ for $\mathrm{C}$ scores and $F(1,69)=18.25, p<.001$ for EC scores. The conditions effect was significant for EC scores, $F(2,69)=3.91, p<.05$, but not $C$ scores. The materials main effect and all interactions were nonsignificant. The performance on each of the four types of trials was nearly the same; on Trials A, B, C, and D the proportions of subjects conserving were .43, $.38, .40$ and .40 with the $C$ criterion and $.29, .28, .28$ and .29 with the EC criterion. This similar performance on the four types of trials was found in each condition.

Table 1 shows the strong order effect in more detail. There is a gradual increase in conservation over the eight trials. The ordering holds up for individual as well as group scores. In a perfectly scaled performance there would be no nonconservation responses on any trials following a conservation response. Of the 25 children who were neither consistent nonconservers nor conservers, 17 had perfect scaling for $\mathrm{C}$ scores and 21 for EC scores. All of the children with imperfect scaling had only one or two trials out of order.

The significant conditions effect for EC scores reflects the fact that there was more conservation in Condition 1 than Condition 2, Scheffé 
TABLE 1

Proportion of Subjects with C and EC Responses on Each

TRIAL With Conditions Combined

\begin{tabular}{ccccccccc}
\hline & \multicolumn{7}{c}{ Trial number } \\
\cline { 2 - 8 } Criteria for conservation & 1 & 2 & 3 & 4 & 5 & 6 & 7 & 8 \\
\hline C & .21 & .31 & .35 & .44 & .43 & .47 & .49 & .51 \\
EC & .18 & .24 & .25 & .28 & .29 & .33 & .35 & .38 \\
\hline
\end{tabular}

$F=9.59, p<.05$, or Condition 3, Scheffé $F=13.53, p<.01$. The mean number of $\mathrm{EC}$ responses in the three conditions was $3.7,1.7$, and 1.4. The superior performance in Condition 1 was expected, but it was also expected that Condition 2 would be easier than Condition 3 . The significant difference for EC scores but not $\mathrm{C}$ scores is related to the fact that $95 \%$ of the conservation responses were supported by adequate explanations in Condition 1, while only 58 and $52 \%$ were in Conditions 2 and 3. This is not surprising because fewer types of explanations were possible in Conditions 2 and 3 than Condition 1. In Conditions 2 and 3 , "previous equality" explanations were not possible and reversibility and addition-subtraction explanations were unlikely. In Condition 1, 57\% of the adequate explanations referred to the fact that nothing was added or taken away and very few referred to counting (10\%). In contrast, the largest category was counting in Conditions $2(49 \%)$, and $3(61 \%)$.

Although correspondence explanations were not frequent, there was some evidence that the more perceptual supports for correspondence, the more frequently correspondence was offered as an adequate explanation. The percentage of correspondence explanations on Trials A, B, C, and D was $29,22,7$, and $7 \%$, respectively.

\section{DISCUSSION}

The results do not support Piaget's claim that provoked correspondence leads to more conservation than spontaneous correspondence does. It does not seem to be true that increasing the link between corresponding elements increases conservation. The superior performance with provoked correspondence in previous studies (Dodwell, 1960; Hood, 1962) was probably due to the methodological problems mentioned earlier.

The equivalent performance on all four levels of correspondence on both sets of materials in three conditions raises the question of whether correspondence is even an important basis for conservation. One could also point to the low percentage of correspondence explanations even 
on the trials with strong perceptual support for correspondence. (Verbal explanations, of course, may not be good indicators of how the child arrived at the conservation answer.) The three conditions, which varied in their emphasis on correspondence, differed only when conservation responses with explanations were the criterion for conservation. Thus, the present study raises doubts about the role of correspondence in the development of conservation. Similarly, in recent years, doubts have been raised about other abilities which Piaget claims underlie the development of conservation, e.g., compensation (Gelman \& Weinberg, 1972) and reversibility (Wallach, 1969).

However, certain aspects of the data suggest that further examination of the correspondence notion might be fruitful. Although the levels of perceptual support for correspondence did not affect conservation, there was a strong order effect $(p<.001)$. There was a large increase in conservation from the first to last trial. Thus, the effect of the supports may have been cumulative rather than specific to each trial. Repeated exposure to correspondence cues could have made the children notice that there ware pairs with no "leftovers." This could lead the children to question their reliance on length and to begin pairing the corresponding objects or counting. Nearly all of the children who switched to conservation continued to conserve for the rest of the session.

The order effect does not seem to be due to a response bias introduced by having several trials with similar questions and procedures. There was not simply an increasing tendency to say "same"; the order effect held up even when adequate explanations were required. It would be difficult to argue that these explanations arose from a response bias. Moreover, the experimenter gave no feedback as to the correctness of the response and was careful not to react differently to nonconservation and conservation answers.

There is another reason for examining the correspondence notion further. The results might seem to contradict the findings of Miller, Heldmeyer, and Miller (1975), Piaget and Morf (cited in Piaget \& Inhelder, 1971), and Whiteman and Peisach (1970) which indicate that perceptual supports for correspondence do facilitate conservation. However, a closer examination of these studies suggests that the number of objects may be a crucial factor. The supports for correspondence are effective only when they operate within a small number of objects. Piaget and Morf found facilitation with rows of four objects but not 12 objects. Miller, Heldmeyer, and Miller and Whiteman and Peisach obtained their facilitation with rows of four and five, respectively. The present study used a larger number of objects (seven) and found no facilitation. Thus, the four levels of support in the present study might have an effect under certain other conditions, i.e., a smaller number of objects.

Further research might examine why a brief experience with the tasks 
in the present study led some children to conservation performance but not others. All of the children who appeared to be nonconservers at the beginning of the session were not equally close to the achievement of conservation performance. Only some of the children were close enough to conservation performance to take advantage of the experience. In fact, using a slightly older population which would be closer to developing conservation might produce an effect of the perceptual supports. There is a need for a precise description of developmental levels within the period of nonconservation.

\section{REFERENCES}

Beilin, H. Stimulus and cognitive transformation in conservation. In D. Elkind \& J. H. Flavell (Eds.), Studies in cognitive development. New York: Oxford University Press, 1969.

Dodwell, C. P. Children's understanding of number and related concepts. Canadian Journal of Psychology, 1960, 14, 191-205.

Gelman R., \& Weinberg, D. H. The relationship between liquid conservation and compensation. Child Development, 1972, 43, 371-383.

Hood, H. B. An experimental study of Piaget's theory of the development of number in children. British Journal of Psychology, 1962, 53, 273-286.

Miller, P. H., Heldmeyer, K. H., \& Miller, S. A. Facilitation of conservation of number in young children. Developmental Psychology, 1975, 11, 253.

Piaget, J. The child's conception of number. New York: Norton, 1952.

Piaget, J., \& Inhelder, B. Mental Imagery in the child. New York: Basic Books, 1971.

Rothenberg, R. B. Conservation of number among four and five year old children: Some methodological considerations. Child Development, 1969, 40, 383-406.

Wallach, L. On the bases of conservation. In D. Elkind \& J. H. Flavell (Eds.), Studies in cognitive development. New York: Oxford University Press, 1969.

Whiteman, M., \& Peisach, E. Perceptual and sensorimotor supports for conservation tasks. Developmental Psychology, 1970, 2, 247-256.

ReCEIVED: July 24, 1975; REVISED: October 13, 1975. 